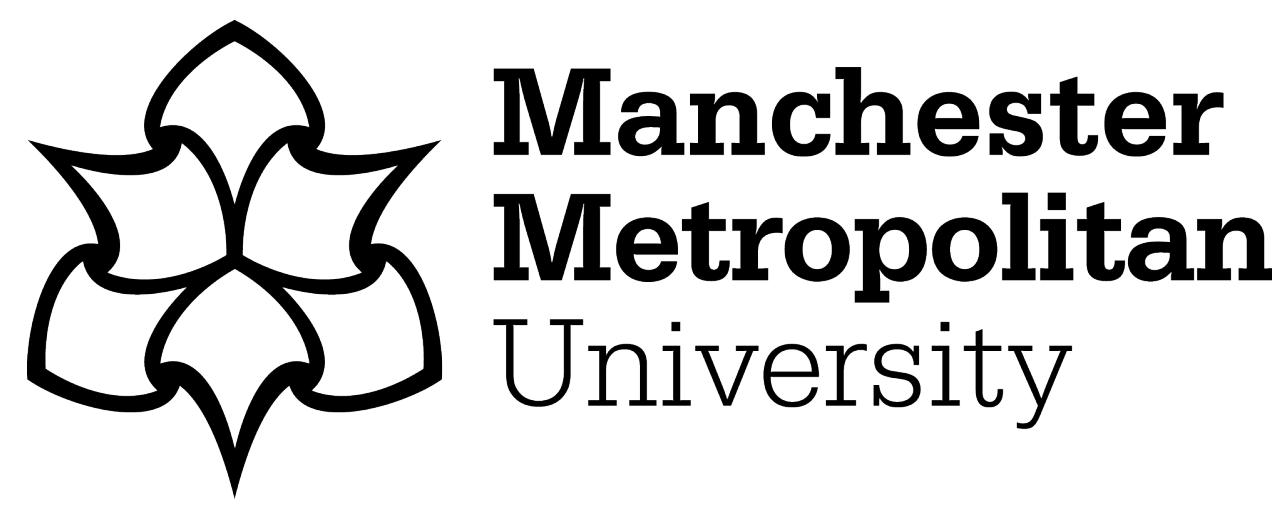

Adebisi, Bamidele, Anoh, K and Rabie, Khaled (2018) An Enhanced Nonlinear Companding Scheme for Reducing PAPR of OFDM Systems. IEEE Systems Journal, 13 (1). pp. 65-75. ISSN 1932-8184

Downloaded from: https://e-space.mmu.ac.uk/621158/ Version: Accepted Version Publisher: Institute of Electrical and Electronics Engineers DOI: https://doi.org/10.1109/JSYST.2018.2851847

Please cite the published version 


\title{
An Enhanced Nonlinear Companding Scheme for Reducing PAPR of OFDM Systems
}

\author{
Bamidele Adebisi, Senior Member IEEE, Kelvin Anoh, Member IEEE and Khaled M. Rabie, Member IEEE
}

\begin{abstract}
A new companding scheme for reducing the peakto-average power ratio (PAPR) of orthogonal frequency-division multiplexing (OFDM) systems is proposed in this study. It proceeds from speech signal processing similar to the earliest $\mu$ law companding (MC) model. The proposed scheme compands (compresses and expands) the amplitudes of OFDM signals to a maximum of 1 - volt. Besides significantly reducing the PAPR, the proposed technique is also able to function as a limiter thus reducing the system complexity and limiting the amplitudes of OFDM symbols to a unity maximum voltage which does not exist in other companding PAPR techniques. Over frequencyselective fading channels with frequency domain equalization and using minimum mean square error (MMSE) to minimize the noise overhead, the proposed technique outperforms four other companding schemes over light and severe fading conditions. Lastly, we demonstrate that PAPR reduction using companding can dispense with correspondingly applying decompanding at the receiver as it amplifies the distortion noise thereby reducing the bit error ratio performance and increases the receiver complexity. We investigated the out-of-band interference of the proposed scheme and also show that it outperforms the other existing techniques by up to $5 \mathrm{~dB}$.
\end{abstract}

Index Terms-Nonlinear companding, orthogonal frequency division multiplexing (OFDM), peak-to-average power ratio (PAPR), limiter, power amplifier (PA), out-of-band interference (OBI).

\section{INTRODUCTION}

$\mathbf{O}$ RTHOGONAL frequency-division multiplexing (OFDM) is a multicarrier scheme for processing communication signals. It has the advantage of high spectral efficiency due to narrow frequencies of OFDM scheme and robustness over multipath fading channels due to increased symbol time. However, its major drawback is the high peak-to-average power ratio (PAPR) problem. The high PAPR may drive the power amplifier (PA) of a communication system to operate near the saturation region. Although nonlinear PAs need large input backoff for linear amplification, increasing the backoff can be used to mitigate the problem. Such solution incurs higher power consumption, thus reducing the efficiency of PAs [1].

Presently, different techniques have been proposed to alleviate the PAPR problem in OFDM systems. These can be broadly grouped into signal distortion techniques, multiple signaling and probabilistic techniques, and coding techniques [2]. Some of these techniques can be performed before or after multicarrier modulation. Based on these two criteria, the three groups of PAPR reduction schemes [2] can further be classified into two groups [3], [4], namely signal scrambling and signal amplitude distortion (SAD) techniques. Examples of PAPR reduction schemes before OFDM modulation include partial transmit sequence [5], Selective Mapping (SLM) [6], [7] and coding [8]. Furthermore, clipping [9]-[11] and companding [12] techniques are applied after OFDM modulation. Other
OFDM design kernels such as wavelets [13] possess low PAPR than conventional OFDM scheme. While clipping expands the system processing time due to $(2 m+1)$ fast Fourier transform (FFT)/inverse FFT (IFFT) operations ( $m$ is the number of clipping/filtering iterations), companding increases the receiver complexity due to decompanding of companded signals.

This paper proposes a nonlinear PAPR reduction technique based on SAD, which does not require decompanding at the receiver, thus relaxing the complexity of using companding techniques. It achieves companding and also limits the amplitude of OFDM symbols to a unit-voltage. Specifically, we introduce in this paper a novel nonlinear companding PAPR reduction technique that can limit both the input and output power levels to a maximum of one (unit-voltage) amplitude. We will refer to this new model as amplitude-limiting companding (ALC) scheme. Such a property makes the proposed scheme more attractive than other previously proposed PAPR reduction schemes. The PAPR and bit error ratio (BER) results of the proposed scheme will be compared with four other widely used companding techniques such as $\mu$-law companding (MC), logbased MC (LMC), hyperbolic arcsin companding (HASC) and exponential companding (EC) schemes [14]-[17].

Companding has potentials to be applied in the candidate waveforms of future 5G standard [18], [19], namely generalized frequency division multiplexing (GFDM). GFDM is a form of filter-bank multicarrier technique for $5 \mathrm{G}$ waveform candidates that deploy standard OFDM structure [18]-[21].

\section{A. Related Work and Summary of Main Contributions}

Companding (an example of SAD PAPR reduction) generally refers to compressing and expanding some OFDM signals amplitudes [14], [15]. It operates on the amplitudes of OFDM signal only without any impact on the phase. The foremost companding model reduces the PAPR of OFDM systems using $\mu$-law [14] and was later explored in other studies [2], [12], [22]-[25]. The $\mu$-law companding (MC) technique is a nonlinear process that involves distorting the amplitudes of some lower OFDM signal amplitudes without impacting the higher amplitude signals which leads to the limited performance of the scheme. However, the ideal companding scheme requires distorting both high and low amplitude signals. Consequently, the seminal work of [15] describes a general model for companding OFDM signals which involves distorting both high and low amplitude signals. Companding has been shown to possess lower in-band distortion noise than clipping [26] and can be applied in power line communication systems [27].

Since the EC method was proposed [15], there are limited novelties afterwards on effective companding models (see [23], [24], [28]-[30]). Only recently, the authors in [31] suggested 
imposing a choice constraint on the default Rayleigh PDF of OFDM signal amplitudes for PAPR reduction. It follows that there are two main models for PAPR reduction using companding scheme, namely $i$ ) using the transformation identity described in [15] by comparing the CDF of unmodified OFDM signal amplitudes and that of a choice uniform distribution model, $i$ i) by imposing a choice constraint on the Rayleigh PDF of OFDM signal amplitudes knowing that $\int_{0}^{c} f_{|x|}(x) d x=1$ where $c$ is a desired cut-off point [31]. Based on these two criteria, especially on the cut-off point, different piecewise companding models [23], [28], [29], [32] have been proposed. Recently, this idea of conversion to uniform distribution has been extended to clipping [10] which is also a post-OFDM PAPR reduction scheme just like companding [25], [26]. There are also the linear companding transforms [33], however, these are not treated in this study as they are outside the scope of our present study.

One problem with previous companding models include the abrupt scaling of input and output signal power levels. In particular, the earliest MC [14] unfairly expands the power level of low amplitude signals thereby leading to unfairly better BER than the unmodified signals [34]. To solve this usually requires additional scaling to normalize the power level [15], [34]. New MC-based models that overcome this problem can be found in [16], [35]. Secondly, most of the PAPR reduction techniques were not discussed over fading channels. Thirdly, these PAPR reduction schemes require decompanding at the receiver which complicates the receiver system. Noting all these complexities, one, we propose the use of a PAPR reduction model that scales both the input and output power of OFDM signal to a desired power level by default. Two, the general system model is tested over multipath fading channel and the results seen to be more robust than all other widely used PAPR models in the literature. Three, we demonstrate that the proposed model can be operated without correspondingly decompanding the transmitted signal due to the fact that the decompanding tool expands both the noise power and also the receiver complexity. As our proposed model can limit the signal to a desired power level, an additional requirement/tool (e.g. limiter) to scale the power of the signal to a desired power level is not required in the proposed PAPR reduction model. Lastly, we derive the decompanding transform to recover the companded signal at the transmitter.

When compared with four other widely known companding techniques namely MC, LMC, HASC and EC, the PAPR and out-of-band interference (OBI) of proposed ALC outperforms those four earlier techniques including the uncompanded signals. Due to the amplitude distortions suffered by the OFDM signals during PAPR reduction, the in-band noise generated by the companding process causes degradation in the BER performances of OFDM systems. Thus, we evaluate the OFDM signals when transmitted over frequency fading channels over light and severe fading conditions. It was observed that the proposed technique is more robust than other techniques over frequency fading channels with minimum mean square error (MMSE) equalization performed in frequency-domain.

The remaining parts of this paper is organized as follows. Section II introduces the system model and describes the non- linear companding transform (NCT). The results and discussion are presented in Section III. Finally, conclusions are drawn in Section IV.

\section{General System Model}

Consider some $N$-point randomly generated data symbols, $\boldsymbol{b}(t)$ $\forall t=1, \cdots, T$. Thus, $\boldsymbol{b}(t)$ is the $t^{t h}$ symbol vector of the form $\boldsymbol{b}(t)=\left[b_{0}, b_{1}, b_{2}, \cdots, b_{N-1}\right]^{T}$, where $[\cdot]^{T}$ represents transpose. The symbols can be processed using the quadrature phase-shift keying (QPSK) or quadrature amplitude modulation (QAM) modulator and then passed through an OFDM modulator to generate some time-domain OFDM symbol as

$$
\boldsymbol{x}(n)=\frac{1}{\sqrt{L N}} \sum_{k=0}^{N-1} \boldsymbol{X}(k) e^{j 2 \pi \frac{k n}{L N}} \forall n=0,1, \cdots, L N-1
$$

where $\boldsymbol{x}(n)$ is the time domain signal and $L$ is the oversampling factor. This factor must satisfy $L \geq 4$ [36]. Since the OFDM symbols are complex, we define their amplitudes as

$$
|\boldsymbol{x}(n)|=\sqrt{\boldsymbol{x}_{r}(n)^{2}+\boldsymbol{x}_{i}(n)^{2}} \quad \forall n=0,1, \cdots, L N-1
$$

where $\boldsymbol{x}_{r}(n)$ and $\boldsymbol{x}_{i}(n)$ are from $\boldsymbol{x}(n)=\boldsymbol{x}_{r}(n)+j \boldsymbol{x}_{i}(n)$ and $j=\sqrt{-1}$. From central limit theorem, the real and imaginary OFDM parts have asymptotically independent and identical Gaussian distribution for any sufficiently large number of subcarriers [3]. In other words, the amplitudes $(|\boldsymbol{x}(n)|)$ follow a Rayleigh distribution as [3]

$$
f_{|\boldsymbol{x}(n)|}\left(x_{0}\right)=\frac{x_{0}^{2}}{\sigma^{2}} \exp \left(-\frac{x_{0}^{2}}{2 \sigma^{2}}\right), \forall n=0,1, \cdots, L N-1
$$

where $x_{0}$ is the discrete-time envelope of $\boldsymbol{x}(n)$ and $\sigma^{2}$ is the variance.

\section{A. Calculating the PAPR}

Now, to estimate the amplitudes of OFDM symbols exceeding a desired amplitude target, $T_{0}$, when processed using a PAPR reduction technique (for instance using an NCT $\mathcal{F}((x))$, the PAPR metric is used. For the original OFDM symbols, $\boldsymbol{x}(n)$, the PAPR can be calculated as

$$
\text { PAPR }=\frac{\max _{n=0,1, \cdots, L N-1}\left(|x(n)|^{2}\right)}{\frac{1}{L N} \sum_{n=0}^{L N-1}\left(|x(n)|^{2}\right)} .
$$

The CDF, $C_{X}$, which is related to the complementary CDF (CCDF) as $C C_{X}=1-C_{X}$ is usually used to measure the PAPR technique performance [16] and is given by

$$
\begin{aligned}
C_{X} & =\operatorname{Pr}\left\{|\boldsymbol{x}(n)| \leq T_{0}\right\} \\
& =1-\exp \left(-\frac{x_{0}^{2}}{\sigma^{2}}\right), \forall x_{0} \geq 0
\end{aligned}
$$

where $C_{X}$ basically measures the probability that the amplitude of the OFDM symbol exceeds a target threshold, $T_{0}$. Considering all points, i.e., $n=0,1, \cdots, L N-1$, the CDF can be rewritten as

$$
\begin{aligned}
C_{X}(\boldsymbol{x}(n)) & =\operatorname{Pr}\left\{|\boldsymbol{x}(0)| \leq T_{0}\right\} \times \operatorname{Pr}\left\{|\boldsymbol{x}(1)| \leq T_{0}\right\} \\
& \times \operatorname{Pr}\left\{|\boldsymbol{x}(2)| \leq T_{0}\right\} \cdots \operatorname{Pr}\left\{|\boldsymbol{x}(L N-1)| \leq T_{0}\right\} \\
& =\left(1-\exp \left(-\frac{x_{0}^{2}}{\sigma^{2}}\right)\right)^{L N}
\end{aligned}
$$


Meanwhile, if the companding function $\mathcal{F}(\boldsymbol{x}(n))=\boldsymbol{y}(n)$, the decompanding function at the receiver is given by $\hat{x}(n)=$ $\mathcal{F}^{-1}(\mathcal{F}(\boldsymbol{x}(n)))$. Then, the PAPR of the companded symbols in this case can be described from the CDF as follows

$$
\begin{aligned}
C_{Y}(\mathcal{F}(\boldsymbol{x}(n))) & =\operatorname{Pr}\left\{|\mathcal{F}(\boldsymbol{x}(n))| \leq T_{0}\right\} \\
& =\operatorname{Pr}\left\{|\boldsymbol{y}(n)| \leq T_{0}\right\}
\end{aligned}
$$

so that the CCDF metric can be written as $C C_{Y}(\mathcal{F}(\boldsymbol{x}(n)))=$ $1-C_{Y}(\mathcal{F}(\boldsymbol{x}(n)))$.

\section{B. Proposed Nonlinear Companding Transform}

The function $\mathcal{F}(\cdot)$ is itself an NCT that compresses/expands the amplitudes of the higher energy signals to a maximum/minimum of a unit-volt, respectively, which lead to significant PAPR reduction. Then, the degree of the distortion with respect to the uncompanded input symbols will influence the BER performance. If $x_{l i n}=\mathcal{G}\{x\}=a_{1} x+b_{1}$ is the output of linearly companded OFDM signal [3], then for NCTs, for example in $\mathrm{MC}$, the function $\log (1+\mu)$ expands the amplitudes of the lower energy symbols [34] only. If an NCT, namely $x_{n l i n}=\mathcal{F}\{x\}=a_{2} x^{\alpha}+b_{2}$ when $1 \leq \alpha \leq \infty$, then $\left|x_{n l i n}(n)\right|^{2}$ increases as $\alpha \rightarrow \infty$ and decreases (in fact, it approaches a constant) as $\frac{1}{\alpha} \rightarrow 0$, where $x$ is the OFDM symbol; this is also upheld in [35]. Based on these, we propose

$$
U=\left(1+\left(\frac{v}{|x(n)|}\right)^{1 / \alpha}\right)^{-\alpha}
$$

where $v$ is used to control the input amplitude of the symbol, $\alpha=\frac{1}{s}$ and $s \in \mathbb{R}^{+}$. Similar to MC motivated by speech processing, (8) has been motivated by the nonlinear behaviour of systems described in [37] and both linear companding transform and NCT discussed in [38]. Considering the phase of the signal [4] (i.e. $\operatorname{sgn}(x)$ ) and the fact that the output amplitude can be limited to a designated voltage level, say $M$, then [37]

$$
y(n)=\mathcal{F}(x(n))=M \times \operatorname{sgn}(x(n)) \times U
$$

where $\operatorname{sgn}(x)=\frac{x}{|x|} \forall x=x_{r}+j x_{i}$ and $M$ is the output limiting value. To ensure that $y(n)$ dissipates equivalent power as the uncompanded symbol (for fair comparison), we weight the result in (9) as

$$
x_{c}(n)=\beta \times \mathcal{F}(x(n))
$$

where $\beta$ is a scaling factor that ensures equal power dissipation with the uncompanded signals, $x(n)$. Define

$$
\mathbb{E}\left\{|x(n)|^{2}\right\}=\int_{0}^{\infty} x^{2} f_{|x(n)|}(x) d x,
$$

where $f_{|x(n)|}$ is the PDF of $x(n)$ usually expressed as in (3), then,

$$
\beta=\left\{\frac{\mathbb{E}\left\{|y(n)|^{2}\right\}}{\mathbb{E}\left\{|x(n)|^{2}\right\}}\right\}^{\frac{1}{2}}
$$

where $\mathbb{E}\{\cdot\}$ is the expectation value operator. For fair comparisons with other PAPR reduction schemes, we also extended the use of $\beta$ normalization parameter in (12) to the other four companding techniques to ensure equal energy dissipation with the
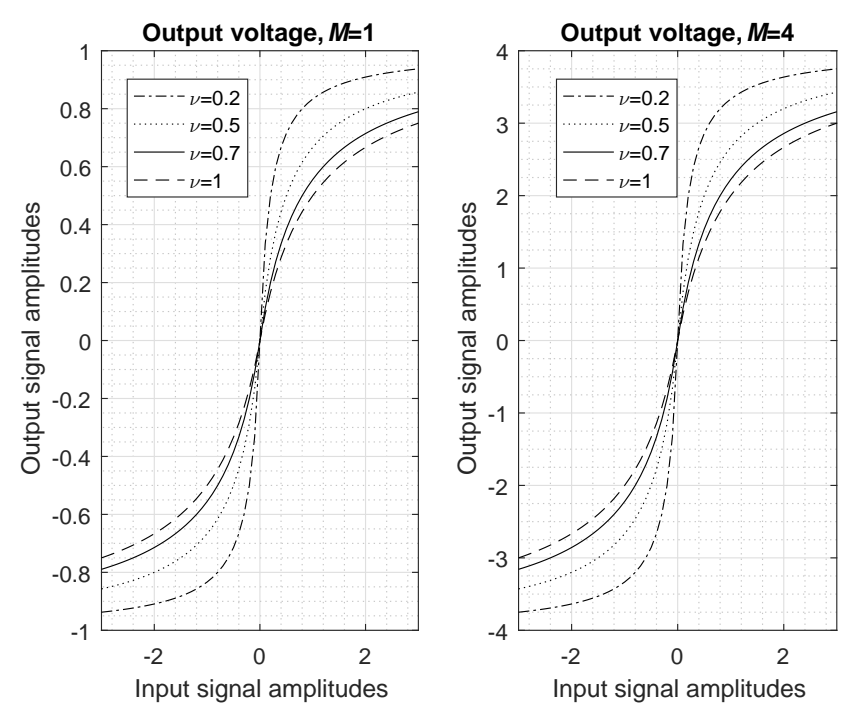

Figure 1. Effects of input voltage and output voltage on the performance of ALC scheme on signal amplitudes

uncompanded OFDM signals. There are $2 L N$ computational operations in the proposed ALC scheme, $7 \mathrm{~N}$ in MC, $13 \mathrm{~N}$ in $\mathrm{EC}$ and $7 \mathrm{~N}$ in LMC, however, there $18 L N$ operations in HASC. In fact, most MC-based models have similar computational costs (see [14], [16], [35]). Although nonlinear in operation, the proposed ALC technique is simpler and more efficient than HASC, EC and LMC (see [14]-[17]).

We show in Fig. 1 the effects of input and output limiting voltage parameters on the signal amplitudes. When the output voltage is $M=1$, all the signal amplitudes are limited to 1 - volt and when increased to $M=4$, the output voltage shows 4 -volts. To ensure a maximum of 1 - volt output signal, we set $M=1$ in all subsequent examples. Also, the PAPR varying parameter $\nu$ limits the input signal according to choice parameter. When small, e.g. $\nu=0.2$ the proposed ALC model distorts the amplitudes by expansion and when large $\nu=1$ our proposed ALC technique distorts the amplitude by compression, however without exceeding the maximum output voltage, $M$.

Since $\mathcal{F}(\cdot)$ can limit all amplitudes to \pm 1 , then $\mathcal{F}(\cdot)$ provides the advantages of a compander and a limiter. From (9), $M$ determines the amplitude of the output signal, $v$ is the input limiting value and $s$ determines the shape of the companded symbol. Since the maximum amplitude of communication signals are usually normalized to unity, we set $0<M \leq 1$. For amplitudes approximable to a unit volt, we suggest $0.5 \leq M \leq 1$. Next, the parameter $v$ determines the PAPR output value and also directly impacts the error vector magnitude (EVM) of the OFDM symbols (see [39]). Similar to the $M$ variable, we suggest also $0.5 \leq v \leq 1$ for amplitudes approximable to a unit volt. There exists poorer BER with further reductions in $v$, for example $v=0.1$, however the PAPR reaches minimum possible. Over an additive white Gaussian noise (AWGN) channel with mean zero and variance $\sigma_{z}^{2}$, then the received signal becomes

$$
\begin{aligned}
\boldsymbol{r}(n) & =\boldsymbol{x}_{c}(n)+\boldsymbol{z}(n) \\
& =\mathcal{F}(\boldsymbol{x}(n))+\boldsymbol{z}(n)
\end{aligned}
$$



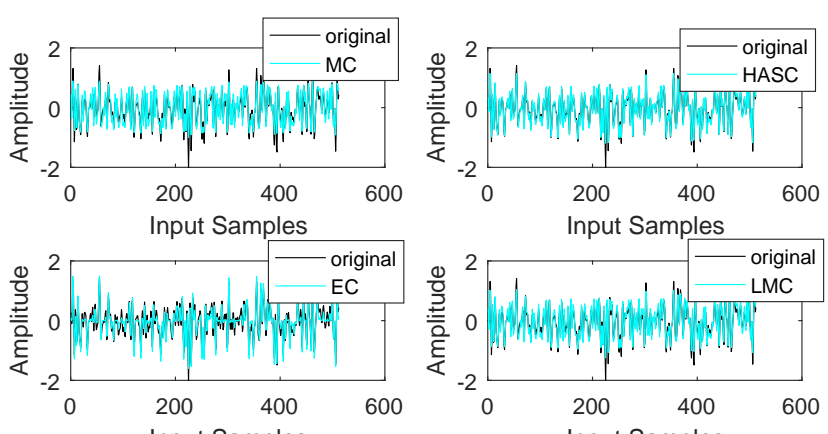

Input Samples

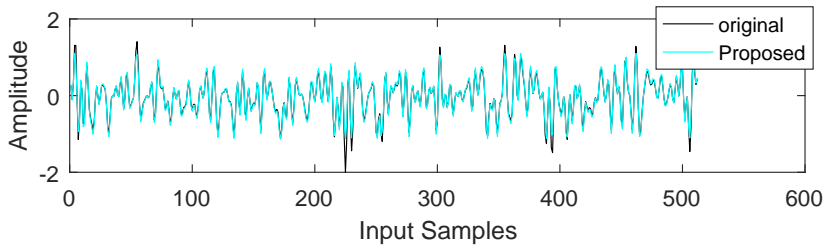

Figure 2. Comparison of original and companded symbols for different methods $(M=1$ and $v=1)$

where $\boldsymbol{z}(n)$ is the AWGN. Let $\gamma=\frac{\mathbb{E}\left\{\left|x_{c}(n)\right|^{2}\right\}}{\mathbb{E}\left\{|z(n)|^{2}\right\}}$ be the received signal-to-noise ratio (SNR). Here, it is worthy to note that any further distortion, by $v$, will increase the background noise thereby reducing the output SNR and thus lowering the BER performance.

Two factors will influence the EVM; one is the degree of distortions to the input symbols which can be controlled in this study by setting $s>0$ and $v>0$. The other is correctly recovering the transmitted symbols by the decompanding function, $\mathcal{F}^{-1}(\mathcal{F}(x(n)))$, at the receiver. Consequently, the decompanding function of the companded OFDM symbol at the receiver can be given by

$$
\begin{aligned}
\mathcal{F}^{-1}(\mathcal{F}(x(n))) & =\frac{v}{\mathcal{B}} \operatorname{sgn}(y(n)) \\
\mathcal{B} & =\left(\left(\frac{M}{|y(n)|}\right)^{s}-1\right)^{\frac{1}{s}} .
\end{aligned}
$$

Proof: See Appendix A for the proof.

We can now rewrite (13) as

$$
\begin{aligned}
\hat{\boldsymbol{x}}(n) & =\mathcal{F}^{-1}(\boldsymbol{r}(n)) \\
& \left.=\mathcal{F}^{-1}(\mathcal{F}(\boldsymbol{x}(n)))+\mathcal{F}^{-1}(\boldsymbol{z}(n))\right) \\
& \left.=\boldsymbol{x}(n)+\mathcal{F}^{-1}(\boldsymbol{z}(n))\right)
\end{aligned}
$$

where $\mathcal{F}^{-1}(\cdot)$ is the decompanding transform. Both $\mathcal{F}(\boldsymbol{x})$ and $\mathcal{F}^{-1}(\mathcal{F}(\boldsymbol{x}))$ are NCTs that are based on $v$ and $M$.

From Bussgang theory [4], [40], the output of the companded signals can be expressed as

$$
\boldsymbol{x}_{c}(n)=\mathcal{F}(\boldsymbol{x}(n))=\rho \boldsymbol{x}(n)+\boldsymbol{d}(n)
$$

where $\rho$ is the attenuation factor that determines the output signal amplitude and $\boldsymbol{d}(n)$ is the distortion noise after $\mathcal{F}(\boldsymbol{x}(n))$ operation. Substituting (16) into (13), gives

$$
\begin{aligned}
\boldsymbol{r}(n) & =\rho \boldsymbol{x}(n)+\boldsymbol{d}(n)+\boldsymbol{z}(n) \\
& =\rho \boldsymbol{x}(n)+\boldsymbol{z}_{a}(n)
\end{aligned}
$$

where the total noise at the receiver can be expressed as

$$
\boldsymbol{z}_{a}(n)=\boldsymbol{d}(n)+\boldsymbol{z}(n) .
$$

At the receiver, using (15) in (17a) the output decompanded signal can be expressed as

$$
\begin{aligned}
\hat{\boldsymbol{x}}(n) & =\mathcal{F}^{-1}(\boldsymbol{r}(n)) \\
& =\boldsymbol{x}(n)-\frac{\boldsymbol{d}(n)}{\rho}+\frac{\boldsymbol{z}(n))}{\rho} \\
& =\boldsymbol{x}(n)+\boldsymbol{z}_{b}(n)
\end{aligned}
$$

where

$$
\left.\boldsymbol{z}_{b}(n)=\frac{1}{\rho} \boldsymbol{z}(n)\right)-\frac{\boldsymbol{d}(n)}{\rho} .
$$

Now, assuming that $\mathcal{F}^{-1}(\boldsymbol{r}(n))$ is absent (in other words, no decompanding is applied) at the receiver, clearly only (17a) is received with $\rho$ impacting the amplitude of $\boldsymbol{x}(n)$ and $\boldsymbol{z}_{a}(n)$. Comparing (18b) and (17b), the noise $\left(\boldsymbol{z}_{a}(n)\right.$ and $\left.\boldsymbol{z}_{b}(n)\right)$ are impacted by $\boldsymbol{d}(n)+\boldsymbol{z}(n)$ and $\left.\frac{1}{\rho} \boldsymbol{z}(n)\right)-\frac{\boldsymbol{d}(n)}{\rho}$ respectively. Since the model in (9) offers both the input and output amplitudes, $\rho=\nu$; this implies that as $\nu \rightarrow 1$, then only the distortion noise $\boldsymbol{d}(n)$ impacts $\boldsymbol{z}(n)$ as in (17b). However, with decompanding at the receiver as in (18b), the distortion noise is amplified by $\rho$ as in $\frac{d(n)}{\rho}$ which diminishes the received signal power (including $\gamma$ ) and further reduces the BER performance. We shall exemplify the impacts decompanding and no decompanding at the receiver in Section III-C.

\section{Impacts of Companding Transform on the Signals}

The degrees of impacts of amplitude distortions on the input symbols during companding are demonstrated in Fig. 2. For the sake of comparison, we also include the behaviours of the LMC, HASC and EC schemes. Comparing the original and companded symbols using the proposed ALC companding model in Fig. 2, it can be observed that all the amplitudes of the input symbols are impacted. Unlike the earlier methods (e.g. MC), the ALC model compands all the amplitudes of the input symbols towards a unit-volt. This will lead to significant PAPR reduction as will be shown later. Notice that LMC, HASC and EC schemes compand all amplitudes but not targeting a maximum/minimum of \pm 1 volt.

\section{Behaviour of the Proposed Nonlinear Compander}

In this section, we compare the companding performance of the proposed transform with EC, MC, HASC and LMC. In Fig. 3 , it can be seen that the MC scheme expands the amplitudes of low amplitude signals only. On the other hand, the proposed ALC scheme compands all the amplitudes to a maximum of $M$-volts $(M=1)$ and a minimum of $\nu$-volts $(\nu=1,0.55)$. Considering the other transforms, LMC largely expands the amplitudes of the OFDM symbols, thus increasing the energy of the output signals which leads to lower BER performance while EC moderately distorts all the amplitudes but larger than the proposed scheme. Worst of all is the HASC scheme which does not alter the amplitudes of lower energy signals, in fact, of all moderately higher energy signals; this will lead to better BER performance. 


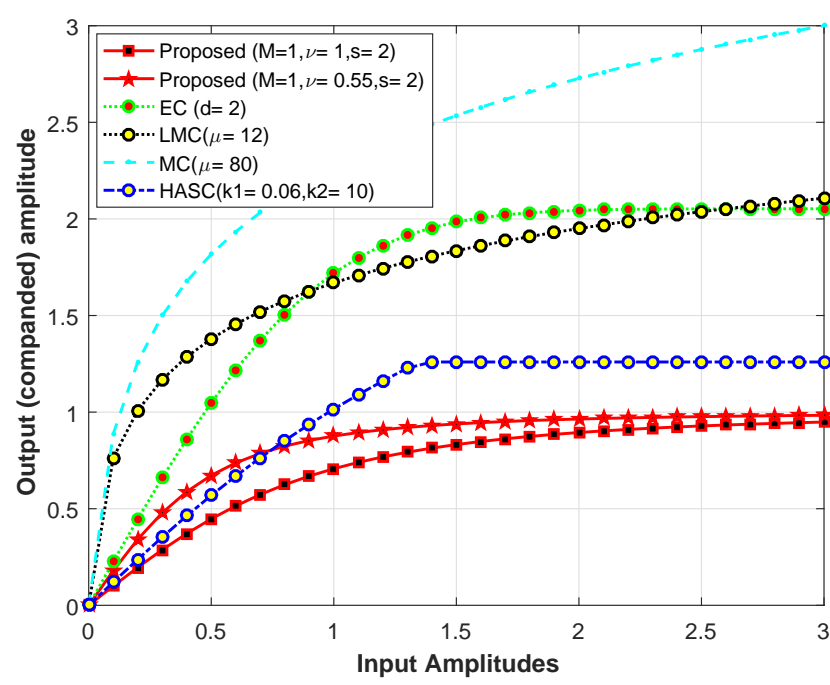

Figure 3. Nonlinear behaviour of proposed compander when $M=1$ in comparison with four other widely used companding models

For any chosen value of $s, \nu$ must be carefully selected not to trade-off the PAPR for the BER or vice-versa. Meanwhile, to achieve much lower PAPR for the proposed ALC technique, $\nu \rightarrow 0$. However, $s$ must be kept reasonably large, for example $1 \leq s \leq \infty$. In Table I, we provide a summary of a comparative performance table for all the PAPR reduction models considered in this study.

\section{E. Transmission and Evaluation of the companded signal over Fading Channel}

The BER performances of PAPR reduction schemes over fading channels are usually scarcely reported. Meanwhile, some authors have exemplified the use of partial transmit sequence (PTS) and OFDM/TDM over fading channels including the effects of nonlinear power amplifiers (PAs) [41], [42]. Due to the difficulty in modeling PAs, PAs are then modeled as typical frequency-selective fading channels models examples in studying PAPR reduction problems [43]. In another study, the filter-bank multicarrier system typical of the future $5 \mathrm{G}$ standard [18]-[20] was studied for PAPR reduction over such fading channel instance [44]. Both [44] and [41] show that over fading channels, multicarrier system suffer severe degradation in BER performance with irreducible error at lower BER measures. These depend on the severity of the fading in the channel and certain PAPR reduction schemes can perform better than others over different fading channel conditions (see Section III-B).

To start with, we model the system as over a Rayleigh multipath fading channel with impulse response $h(\tau)$ and the channel transfer function $H(f)$, where

$$
H(f) \underset{I F F T}{\stackrel{F F T}{\rightleftharpoons}} h(\tau) .
$$

Generally, channel modeling may involve perfect channel state information (CSI) or imperfect CSI. In time division duplexing (TDD), the CSI is simplified due to the reciprocity of the channel however, in frequency division duplexing (FDD), a feedback mechanism is required to update the transmitter with
CSI observed at the receiver. This is usually encumbered by i) noise in CSI sent to transmitter causing mismatch and $i$ ) the CSI is usually outdated due to finite delay associated with feedback [45], [46]. To relax these complexities, we assume that the CSI is perfectly known at the receiver. At the receiver, the received signal (after the removal of cyclic prefix) can be expressed as

$$
Y(f)=H(f) X_{c}(f)+Z(f), \quad \forall f=0,1, \cdots, L N-1
$$

where $X_{c}(f)$ and $Z(f)$ are the transformed companded signal and the additive white Gaussian noise, respectively, in frequency domain. Given the frequency-selective fading channel, the new SNR can be expressed as

$$
\gamma\left(X_{c}, H\right)=\frac{\mathbb{E}\left\{\left|H(f) X_{c}(f)\right|^{2}\right\}}{\mathbb{E}\left\{|Z(f)|^{2}\right\}}=\frac{\sigma_{x, H}^{2}}{\sigma_{Z}^{2}}
$$

where $\sigma_{x, H}^{2}$ is the received signal power over fading channel, $H \sim \mathcal{N}\left(\mu_{H}, \sigma_{x, H}^{2}\right)$ and $\sigma_{Z}^{2}$ is from $Z \sim \mathcal{N}\left(0, \sigma_{Z}^{2}\right)$.

At the receiver, the received signal $Y(f)$ is then decompanded to recover the estimate of the original transmitted signal before PAPR mitigation processing. We also similarly performed decompanding for other companding models (EC, LMC, HASC, MC) to which the proposed ALC is compared with. From (21), the BER performance depends on $\sigma_{Z}^{2}$. To minimize its effect and also compensate for the effect of $H$, we construct a MMSE filter which has been widely adopted in noise-sensitive communication systems [47]. Using the MMSE filter, the received signal can be expressed as

$$
\hat{X}_{c}=\mathcal{G}_{M M S E}(f) Y(f)
$$

where the $f$ index shows the frequency indices of the channel coefficient and $\mathcal{G}_{M M S E}(f)$ represents the frequency domain MMSE filter which can be expressed as [42]

$$
\mathcal{G}_{M M S E}(f)=\frac{H^{*}(f)}{\left|H^{*}(f)\right|^{2}+\left(\frac{E_{s}}{N_{0}}\right)^{-1}}
$$

where $E_{s} / N_{0}$ is the output SNR. Since the MMSE scheme has potential to improve the BER performance by reducing the noise, the output received signal estimate can be expressed as

$$
\begin{aligned}
\hat{X}_{c} & =\mathcal{G}_{M M S E}(f) Y(f) \\
& =X_{c}+\frac{H^{*}(f)}{\left|H^{*}(f)\right|^{2}+\left(\frac{E_{s}}{N_{0}}\right)^{-1}} Z(f) \\
& =X_{c}+\hat{Z}
\end{aligned}
$$

where

$$
\hat{Z}=\frac{H^{*}(f)}{\left|H^{*}(f)\right|^{2}+\left(\frac{E_{s}}{N_{0}}\right)^{-1}} Z(f)
$$

from which the characteristic covariance matrix can be used to discuss the output SNR and BER performance. In the case of AWGN-only channel, the companded signal was impacted by the noise from the companding process and the AWGN, however, over fading channel the BER of the signal will further be impacted by $\mathcal{G}_{M M S E}(f)$. Examples of these results have been reported involving the use of PAPR and MMSE in [41], [42], thus we adopt these in the results in Section III-B. 
Table I

COMPARATIVE ANALYSES OF DIFFERENT COMPANDING PAPR REDUCTION MODELS

\begin{tabular}{|c|c|l|c|c|c|c|}
\hline $\begin{array}{c}\text { Companding } \\
\text { model }\end{array}$ & $\begin{array}{c}\text { Shape } \\
\text { parameter }\end{array}$ & $\begin{array}{c}\text { Power level } \\
\text { normalization }\end{array}$ & Output amplitude parameter & $\begin{array}{c}\text { Expands weak } \\
\text { signal }\end{array}$ & $\begin{array}{c}\text { Compress } \\
\text { stronger signals }\end{array}$ & $\begin{array}{c}\text { Limits input/output } \\
\text { power level }\end{array}$ \\
\hline \hline EC & $d>0$ & Inbuilt & No & $\begin{array}{c}\text { When } d \text { is } \\
\text { large }\end{array}$ & Always & No \\
\hline MC & $\mu>0$ & Required & No & Always & No & No \\
\hline LMC & $\mu, a, b>0$ & Inbuilt & No & Always & Always & No \\
\hline ALC & $s>0$ & Inbuilt & Yes, $M=1$ & Always & Always & Yes, $M=1, \nu=1$ \\
\hline HASC & $c, k_{1} k_{2}>0$ & Inbuilt & No & Slightly & Always & No \\
\hline
\end{tabular}

\section{Results AND Discussions}

In the simulations, we generate $N=128$ random input symbols and mapped these using QPSK modulation, then normalized the signals to ensure a maximum output power of 1 . The resulting symbols are oversampled $L=4$-times so that an oversized IFFT/FFT of 512 points is used to generate OFDM time domain signals as shown in (1). To protect the signals over fading channels, cyclic prefix which has $25 \%$ length of the time-domain signal is added. Next, we estimate the PAPR for the original symbols before applying the proposed ALC PAPR reduction scheme. Later, we measure the PAPR of the proposed ALC PAPR reduction scheme to assess level of reduction achieved compared to the original OFDM symbols. Subsequently, the PAPR reduced OFDM signals are passed through the channel and AWGN is added. At the receiver, we apply the inverse ALC operation to decompand the companded signals. Then we removed the cyclic prefix and performed the forward FFT to transform the received signal into frequency domain. In the frequency domain, we apply the MMSE equalization described in (24a) and performed inverse oversampling. The received signal were demodulated using QPSK before bit error estimation. We present the results in Sections III-A and III-B. These processes are then repeated for HASC, LMC and EC techniques for comparisons. In all we adopt $s=60$ and $M=1$.

\section{A. Performance Evaluation of the Proposed Scheme over AWGN-only Channels}

Fig. 4 shows the results of the proposed ALC scheme and the other four companding schemes under consideration. Although all the companding schemes reduce the PAPR, some outperform others. However, comparing the proposed ALC scheme with LMC at $10^{-5} \mathrm{CCDF}$, it is found that although the proposed scheme outperforms all other (LMC, HASC, MC and EC) schemes at $10^{-5}$ CCDF the proposed ALC scheme is specifically better than LMC by up to $3.3 \mathrm{~dB}$ when $\nu=0.7$. When $v=1$, the proposed scheme also reduces the PAPR of the original symbol from $12 \mathrm{~dB}$ to $4.6 \mathrm{~dB}$ and outperforms three of the other schemes (EC, HASC and LMC), but slightly worse than MC and LMC at $10^{-5} \mathrm{CCDF}$.

In addition, the proposed technique achieves the lowest BER among all the existing schemes under investigation but slightly worse than the uncompanded signals as it is found in Fig. 5 when $v=1$. This can be explained on the premise that our proposed ALC scheme induces the least distortion noise to the signals when companded than other companding schemes. Specifically, at $10^{-4}$ BER, the proposed scheme gains $5 \mathrm{~dB}$ in

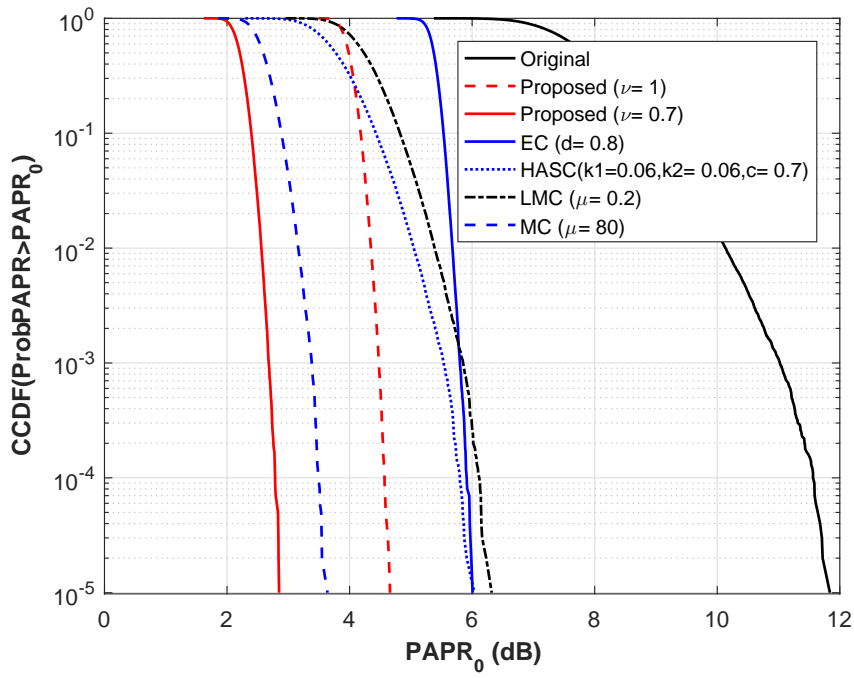

Figure 4. PAPR comparison of the proposed method with other companding techniques (when $M=1, N=128, L=4$ ) over AWGN fading channel.

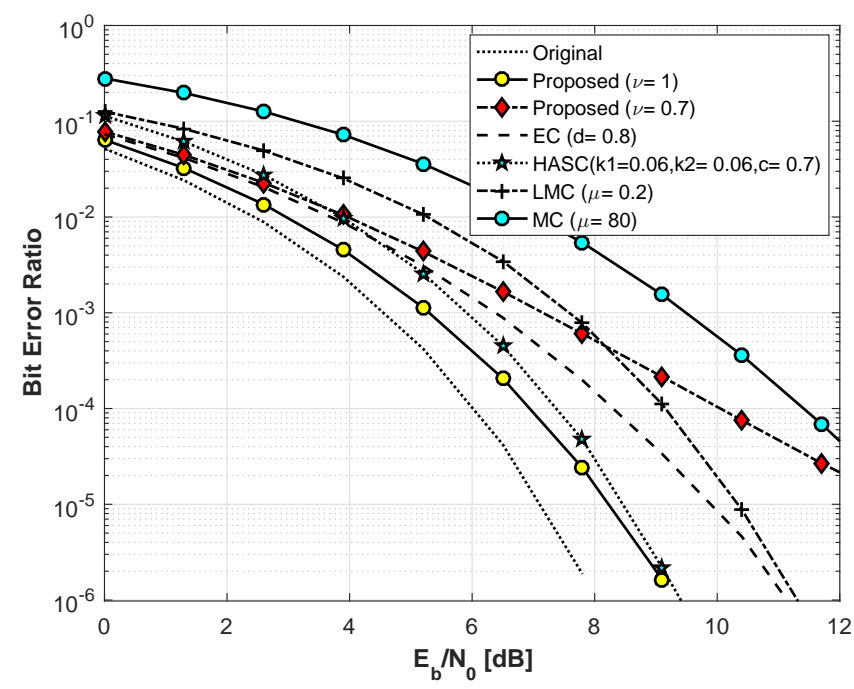

Figure 5. BER performance of different companding techniques with the proposed method ( $M=1, N=128, L=4)$

SNR better than MC, $2 \mathrm{~dB}$ better than LMC, $1 \mathrm{~dB}$ better than EC and slightly better than HASC.

Recall in Section II-D, we showed that LMC largely expands the amplitudes of the OFDM symbols, thus increasing the energy of the output signals which leads to lower BER performance better than MC, meanwhile the proposed ALC scheme 


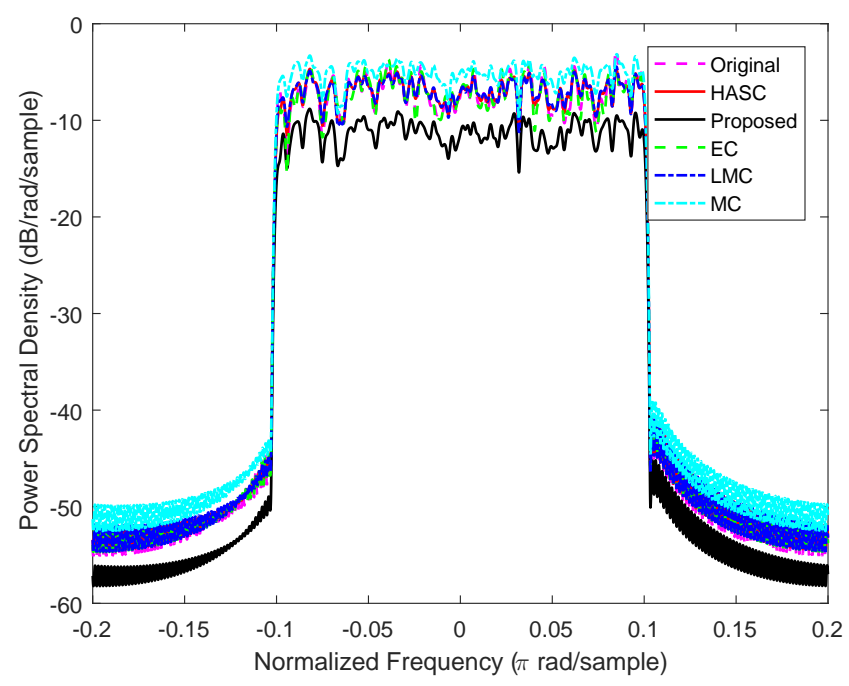

Figure 6. PSD comparisons of the different companding PAPR reduction techniques including the uncompanded OFDM signals.

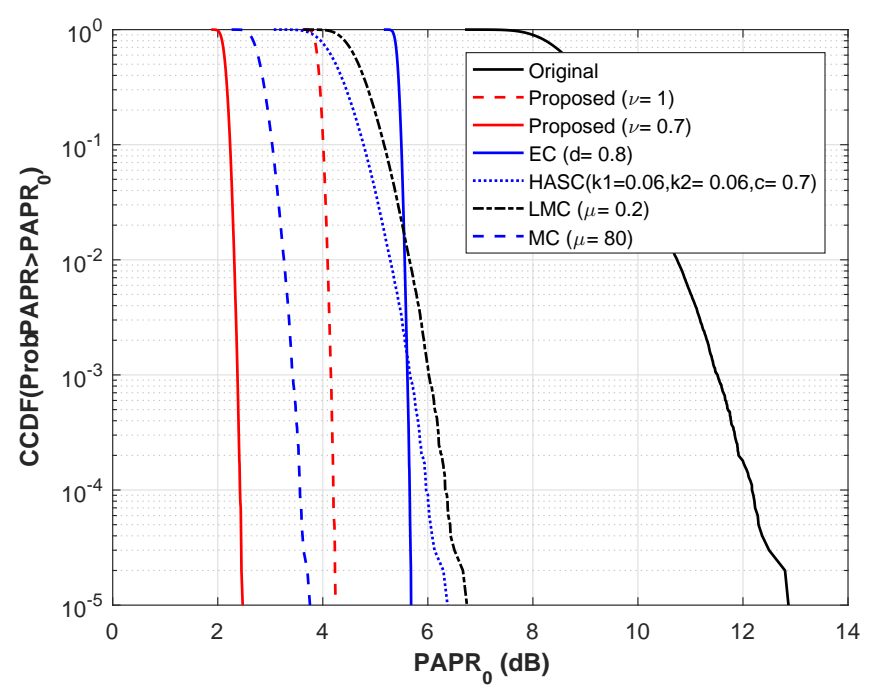

Figure 7. PAPR comparison of the proposed method with other companding techniques (when $M=1, N=512, L=4$ )

moderately expands the energy of the lower amplitude signals and compresses the amplitudes of high energy signals which was instrumental to both good PAPR and BER performances. Also in Fig. 5 when $v=0.7$, in other words, when the attenuation is increased the amplitudes of the symbols is then largely distorted leading to more distortion noise and thus lowering the BER performance. Notwithstanding, the proposed ALC scheme outperforms MC, LMC and HASC at low BER measure.

Finally, we explore OBI and also plot in Fig. 6 the power spectral density (PSD) for the five companding techniques including that of the original OFDM symbols. Clearly, the proposed ALC technique produces $5.64 \mathrm{~dB}, 3.53 \mathrm{~dB}, 3.49 \mathrm{~dB}$, and $3.66 \mathrm{~dB}$ OBI lower than the MC, LMC, EC and HASC techniques, respectively, including a $3.1 \mathrm{~dB}$ better than the uncompanded signals.

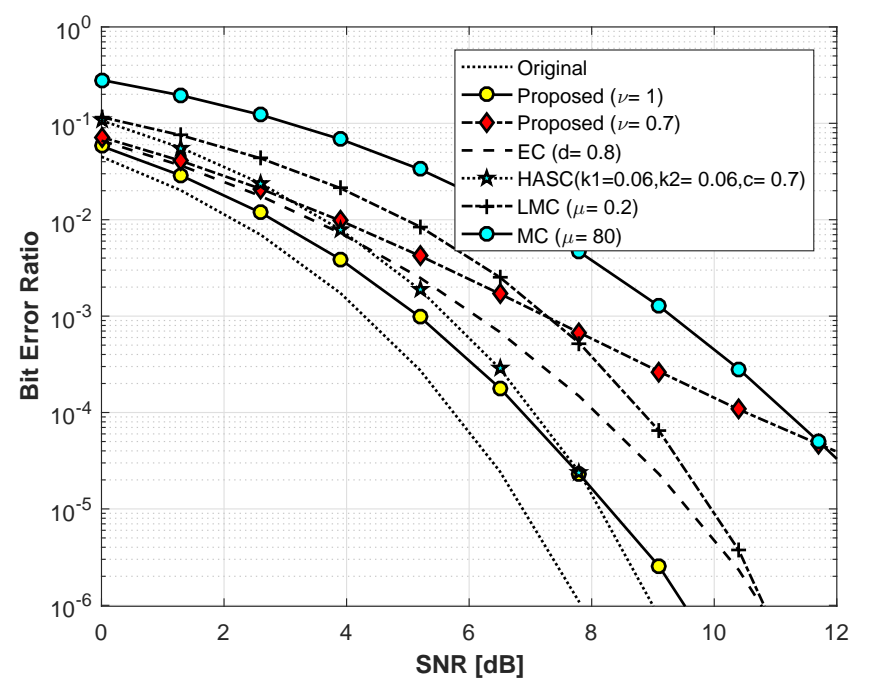

Figure 8. BER performance of different companding techniques with the proposed ALC method ( $M=1, N=512, L=4)$ over AWGN fading channel

It is worth noting that it is possible to completely reduce the PAPR further using the proposed model by further reducing the PAPR determining value $\nu$, however, this will further diminish the BER performance. To further illustrate the superior performances of our results, we demonstrate the performance of the proposed scheme with the four other companding schemes under discussion as shown in Figs. 7 and 8 for PAPR and BER performances when $N$ is increased. As in Figs. 4 and 5 , our proposed ALC scheme outperforms the other schemes upholding the superiority of our model both when $N$ is large or small.

\section{B. Performance Evaluation of Proposed Companding Scheme over Frequency-Selective Fading Channels With MMSE}

Although not commonly reported, we examine the BER performance of the proposed companding transform over frequency-selective fading channel. The resulting companded signal is linearly convolved with the channel impulse response in time-domain and AWGN is added. The AWGN noise impacts the in-band distortions already incurred by the signals during compression and expansion of the amplitudes. At the receiver, the received signal is decompanded and then demodulated using OFDM demodulator. This is done by the removal of cyclic prefix followed by performing the FFT on the received signal which transforms the signal into frequency domain and the results are shown in (20). In Fig. 9, we depict the impulse and channel responses of the channel model used.

Now, with the received signal $Y(f)$ in frequency domain, and using perfect knowledge of CSI at the receiver, frequency domain equalization is performed as in (22) and the results are shown in Fig. 10. In Fig. 10, the BER performances of the five companding techniques under study are compared over a frequency-selective fading channel with 2 and 5 channel taps respectively. It is found that the proposed ALC technique outperforms the other four techniques under investigation in terms of BER. 

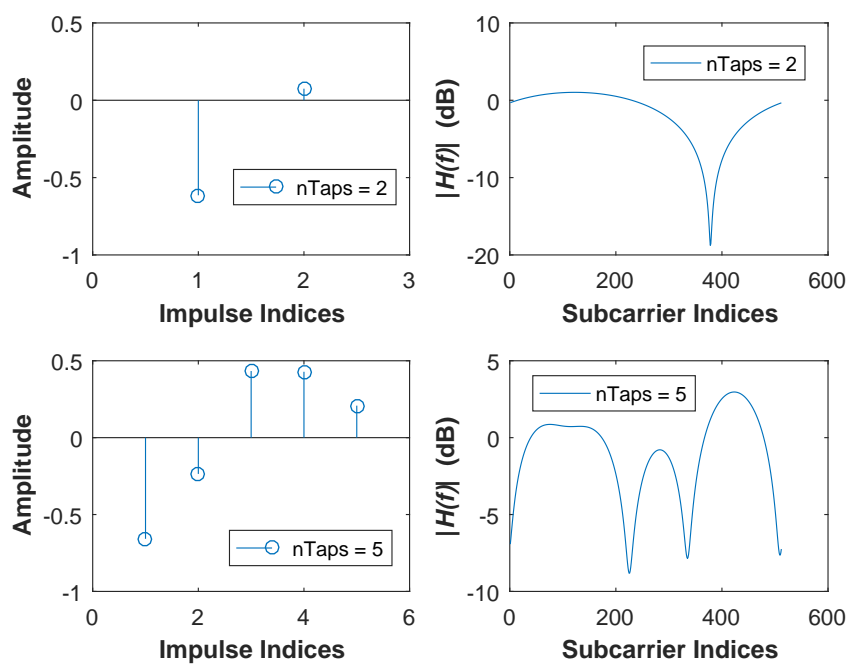

Figure 9. Channel description and behaviour in both time and frequency domains

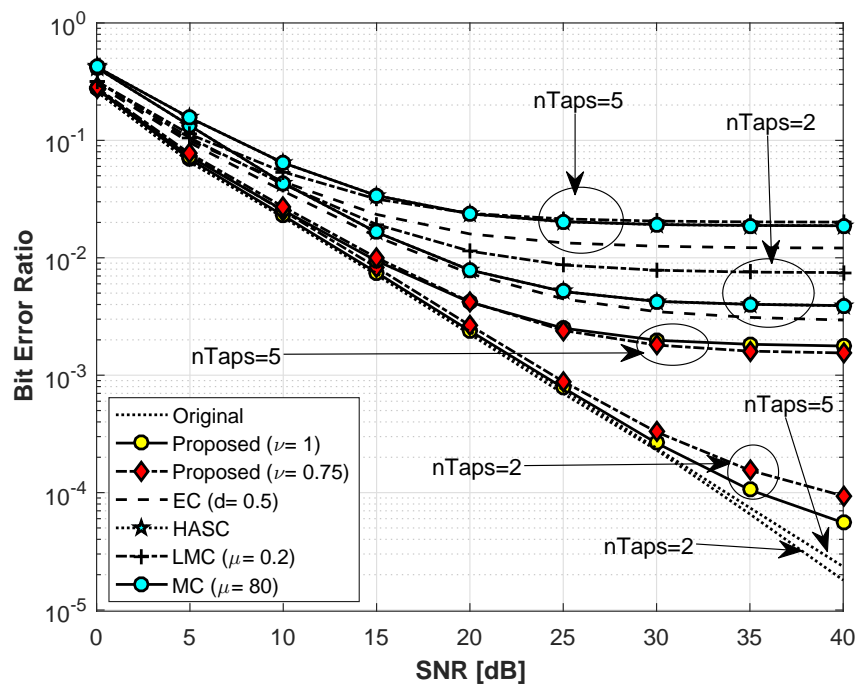

Figure 10. BER performance evaluation of proposed companding scheme over frequency-selective fading channel with MMSE equalization method in comparison with other companding schemes, namely, MC, LMC, EC, HASC (number of channel taps (nTaps $=2$ and 5), $L=4, N=128$ )

Companding involves deliberately distorting the amplitudes of OFDM signals. This amplitude distortion creates in-band distortion noise that impacts the error vector magnitudes of the originally transmitted data symbols. EVM measure the deviation of processed signals (e.g. amplitude distorted symbols) from the original constellation position which in turn gives information into the possible BER performance of the system. Although a PAPR technique that significantly reduces PAPR of OFDM signals is attractive, the BER must also be preserved. At significantly low BER in Fig. 10, say $10^{-5}$, the ALC companded OFDM signals slightly deteriorated as the channels taps increased from 2 to 5 . On the other hand, the ALC scheme performed similarly as the uncompanded signals until the significantly low BER is achieved. This shows its robustness over frequency-selective fading channels after being

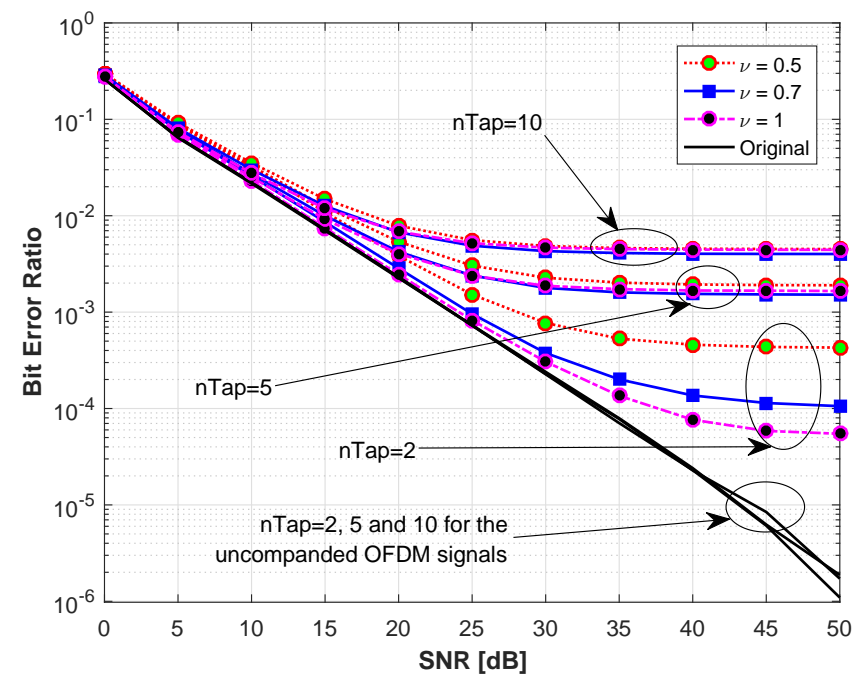

Figure 11. BER performance evaluation of proposed companding scheme over frequency-selective fading channel with MMSE equalization method in comparison with other companding schemes, namely, MC, LMC, EC, HASC for high amplitude distortion over severer channel fading $(L=4, N=128$, number of channel taps (nTaps $=2,5,10$ ) and $\nu=0.5,0.7$ and 1 volts)

impacted by fading, for nTap $=2$, by the in-band distortion noise due to amplitudes companding $(\rho)$. Now, compared to EC, LMC, HASC and MC schemes, the proposed ALC scheme outperformed all. The trend of performance of the proposed technique has been derived from the fact that it compands OFDM signals to a preset amplitude threshold allowing uniform spread of the channel fading thus leading to lower BER. Also, when the MMSE equalization filter impacts the distortion noise and AWGN at the receiver, higher distortion noise leads to poorer BER performance. When increased to 5 taps, the EC, LMC, HASC and MC schemes were severely impaired at lower BER measures than the proposed scheme.

In Fig. 11, we demonstrate the performance of the proposed scheme over fading channels when the attenuation increases and under severe fading conditions (e.g. nTaps $=2,5$ and 10). It is found that increasing the attenuation produced slight impairments compared to increasing fading channel conditions, nTaps $=10$. Thus, we infer that despite the PAPR reduction ability provided by the proposed ALC scheme, it is also robust over severe fading conditions.

\section{Performance Evaluation of Decompanded Systems and Un- decompanded Systems}

We showed in Section II-B that decompanding at receiver amplifies the noise (both distortion noise and AWGN). In this section, we show the performances of the systems with and without decompanding at the receiver for fading and non-fading channels.

From Fig. 12, the results show that decompanding degrades the BER performance at the receiver. In addition, increasing the attenuation weight also further increases the distortion noise which is amplified if decompanded. Over fading channels as shown in Fig. 13, the equalization filter amplifies the distortion with and without the decompanding. 


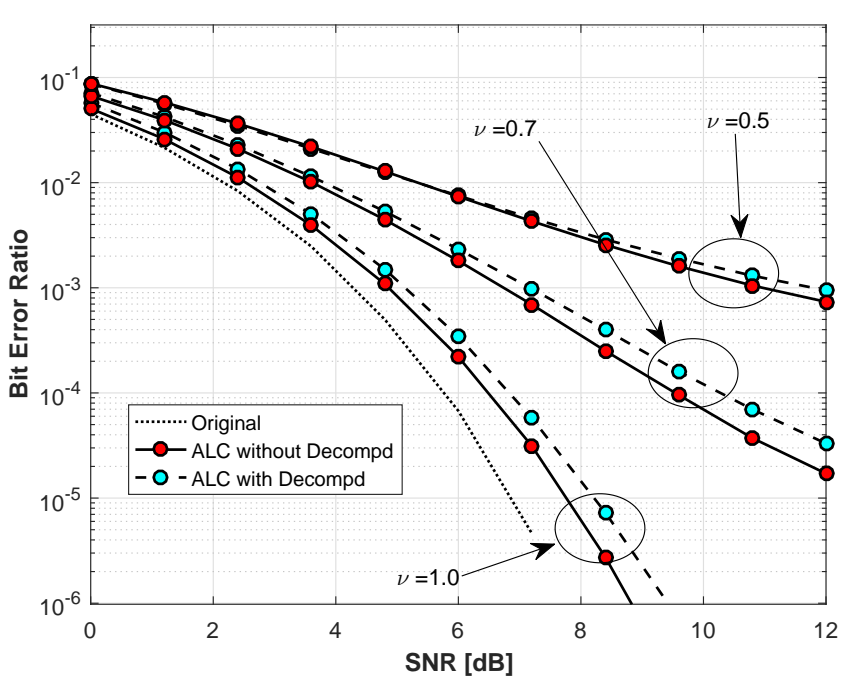

Figure 12. BER performance evaluation companded signals with and without decompanding for different attenuation weights over AWGN-only channel

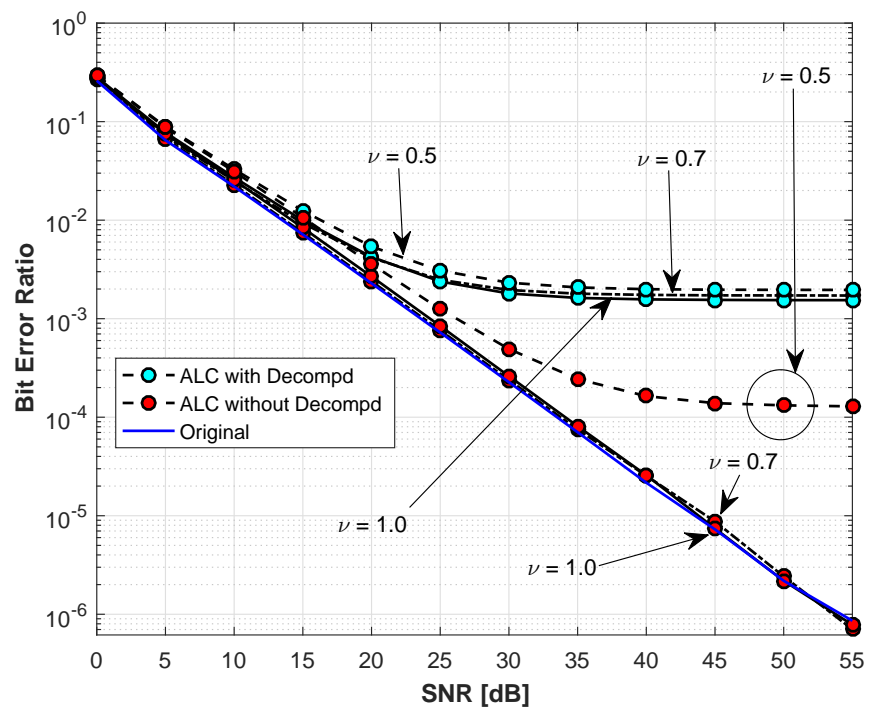

Figure 13. BER performance evaluation companded signals with and without decompanding for different attenuation weights over frequency-selective fading channel with AWGN

However, the impact becomes more significant when decompanding is applied at the receiver. Thus, companding PAPR reduction schemes for reducing the PAPR of OFDM signals should be implemented without decompanding as it both degrades the BER performance of the system and also increases the receiver complexity, expands processing time and consumes system power.

\section{CONCLUSION}

In this paper, we presented a new companding technique that has the ability to reduce PAPR as well as limit the peak amplitudes of OFDM symbols to a unit-voltage. Using simulation results, the proposed method was shown to outperform four other widely used companding schemes, such as MC, HASC, LMC and EC methods in terms of PAPR, OBI and BER metrics over AWGN channel. Based on the desired output performance of the OFDM system, the PAPR can be completely eliminated by further resetting the PAPR determining parameter, $\nu$ in the proposed model, to values smaller than 0.5 volts. Over fading channels, it is found that the proposed technique significantly outperforms EC, LMC, HASC and MC schemes at all channel tap instances thus making it more suitable for applications over practicable real-life channel environments. In fact, at less severe fading channel conditions such as the Rician channel or lineof-sight transmissions, it can be conjectured that the proposed companding scheme will perform as similar to dispensing without PAPR reduction techniques leading to low complexity systems. It was further showed that companding PAPR schemes can be deployed without correspondingly decompanding the received signals at the receiver as the decompanding process amplifies the distortion noise by the attenuation weight thus leading to poorer BER performances. Also, operating companding schemes in OFDM system transmitters only can as well reduce the receiver complexity and eliminate expending the required system power to process the decompanding process.

\section{APPENDIX A}

Define $\operatorname{sgn}(y)=\frac{y}{|y|}$ and let $\operatorname{sgn}(x) \approx \operatorname{sgn}(y)$, then rearranging (9), we obtain

$$
\begin{aligned}
\frac{y}{\operatorname{sgn}(x)} & =\frac{M}{\left(1+\left(\frac{v}{|x|}\right)^{s}\right)^{\frac{1}{s}}} \\
\left(1+\left(\frac{v}{|x|}\right)^{s}\right)^{\frac{1}{s}} & =\frac{M}{|y|} \\
\left(\frac{v}{|x|}\right)^{s} & =\left(\frac{M}{|y|}\right)^{s}-1 \Longrightarrow|x|=\frac{v}{\mathcal{B}}
\end{aligned}
$$

where $\mathcal{B}=\left(\left(\frac{M}{|y|}\right)^{s}-1\right)^{\frac{1}{s}}$. We have assumed that $\operatorname{sgn}(x) \approx$ $\operatorname{sgn}(y)$ because companding transform only operates on the amplitudes of OFDM signals, however, it does not impact the phase of the signal which $\operatorname{sgn}(x)$ and $\operatorname{sgn}(y)$ represent. Multiplying through by $\operatorname{sgn}(x)$,

$$
x=\operatorname{sgn}(y) \frac{v}{\mathcal{B}}
$$

so that (where $\operatorname{sgn}(x) \approx \operatorname{sgn}(y))$

$$
\begin{aligned}
\mathcal{F}^{-1}(\mathcal{F}(x)) & =\operatorname{sgn}(y) \frac{v}{\mathcal{B}} \\
\mathcal{B} & =\left(\left(\frac{M}{|y|}\right)^{s}-1\right)^{\frac{1}{s}} .
\end{aligned}
$$

This is the end of the proof.

\section{REFERENCES}

[1] X. Zhu, W. Pan, H. Li, and Y. Tang. Simplified approach to optimized iterative clipping and filtering for PAPR reduction of OFDM signals. IEEE Trans. Commun., 61(5):1891-1901, 2013.

[2] Y. Rahmatallah and S. Mohan. Peak-To-Average Power Ratio Reduction in Ofdm systems: A Survey And Taxonomy. IEEE Commun. Surveys Tut., 15(4):1567-1592, Fourth Quarter 2013.

[3] S. Sengar and P. Bhattacharya. Performance improvement in OFDM system by PAPR reduction. arXiv preprint arXiv:1205.2269, 2012.

[4] J. Hou, J. Ge, D. Zhai, and J. Li. Peak-to-Average Power Ratio Reduction of OFDM Signals With Nonlinear Companding Scheme. IEEE Trans. Broadcast., 56(2):258-262, Jun. 2010. 
[5] C. Weng, C. Chang, C. Chen, and H. Hung. Novel low-complexity partial transmit sequences scheme for PAPR reduction in OFDM systems using adaptive differential evolution algorithm. Wireless Personal Commun., pages 1-16, 2013.

[6] A. Boonkajay, T. Obara, T. Yamamoto, and F. Adachi. Selective mapping for broadband single-carrier transmission using joint tx/rx mmse-fde. In 2013 IEEE 24th Int. Symp. PIMRC, pages 641-645, 2013.

[7] E. Hassan, Z. Xu, S. El-Khamy, M. Dessouky, S. El-Dolil, and F. Abd El-Samie. Peak-to-average power ratio reduction using selective mapping with unequal power distribution. J. Central South University, 19(7):19021908, 2012

[8] D. Qu, L. Li, and T. Jiang. Invertible subset LDPC code for PAPR reduction in OFDM systems with low complexity. IEEE Trans. Wireless Commun., 13(4):2204-2213, Apr. 2014.

[9] J. Armstrong. Peak-to-average power reduction for OFDM by repeated clipping and frequency domain filtering. Electron. Lett., 38(5):246-247, Feb. 2002.

[10] K. Anoh, C. Tanriover, B. Adebisi, and M. Hammoudeh. A new approach to iterative clipping and filtering PAPR reduction scheme for OFDM systems. IEEE Access, 6:17533-17544, Sept. 2017.

[11] K. Anoh, C. Tanriover, and B. Adebisi. On the optimization of iterative clipping and filtering for PAPR reduction in OFDM systems. IEEE Access, 5:12004-12013, Jun. 2017.

[12] K. Bandara, A. Sewaiwar, and Y. H. Chung. Efficient nonlinear companding scheme for substantial reduction in peak-to-average power ratio of OFDM. Journal of Systems Engineering and Electronics, 26(5):924931, Oct. 2015.

[13] K. Anoh, A. Ikpehai, K. Rabie, B. Adebisi, and W. Popoola. PAPR reduction of wavelet-OFDM systems using pilot symbols. In IEEE ISPLC, 2018

[14] X. Wang, T. Tjhung, and C. Ng. Reduction of peak-to-average power ratio of OFDM system using a companding technique. IEEE Trans. Broadcast., 45(3):303-307, 1999

[15] T. Jiang, Y. Yang, and Y. Song. Exponential companding technique for PAPR reduction in OFDM systems. IEEE Trans. Broadcast., 51(2):244248, 2005.

[16] O. Gazi. A new companding technique for PAPR reduction in OFDM communication systems. In 2011 3rd Intl. Congress on Ultra Modern Telecom. Control Syst. Workshops (ICUMT), pages 1-5, Oct. 2011.

[17] Y. Wang, L. h. Wang, J. h. Ge, and B. Ai. Nonlinear companding transform technique for reducing PAPR of OFDM signals. IEEE Trans. Consum. Electron., 58(3):752-757, Aug. 2012.

[18] Z. Sharifian, M. Omidi, A. Farhang, and H. Saeedi-Sourck. Polynomialbased compressing and iterative expanding for PAPR reduction in GFDM. In 23rd Iranian Conf. Elect. Engr., pages 518-523, 2015.

[19] B. Farhang-Boroujeny and H. Moradi. OFDM Inspired Waveforms for 5G. IEEE Commun. Surveys Tuts., 18(4):2474-2492, Fourthquarter 2016.

[20] L. Cho, C. H. Ting, C. Y. Chen, C. H. Yau, and C. Y. Hsu. When UUDN meets 5G waveforms: New challenge of signal linearity? In 2017 International Conference on Applied System Innovation (ICASI), pages 770-773, May 2017.

[21] X. Zhang, L. Chen, J. Qiu, and J. Abdoli. On the waveform for 5G. IEEE Commun. Mag., 54(11):74-80, Nov. 2016.

[22] T. Jiang and Y. Wu. An Overview: Peak-to-Average Power Ratio Reduction Techniques for OFDM Signals. IEEE Trans. Broadcast., 54(2):257-268, Jun. 2008

[23] H. Zhang, L. L. Yang, and L. Hanzo. Piecewise companding transform assisted optical-ofdm systems for indoor visible light communications. IEEE Access, 5:295-311, Feb. 2017.

[24] Y. Wang and L. Wang. Transforming the Statistical Distribution of Wireless OFDM Signal for PAPR Reduction. Wireless Personal Commun., pages 1-13, 2017.

[25] K. Anoh, B. Adebisi, K. Rabie, M. Hammoudeh, and H. Gacanin. On Companding and Optimization of OFDM Signals for Mitigating Impulsive Noise in Power-line Communication Systems. IEEE Access, 5:21818 - 21830, Sept. 2017.

[26] K. Anoh, B. Adebisi, and M. Hammoudeh. A Comparison of ICF and Companding for Impulsive Noise Mitigation in Powerline Communication Systems. In Int. Conf. Future Netw. Distributed Syst., Jul.. 2017.

[27] B. Adebisi, K. Anoh, K. M. Rabie, A. Ikpehai, M. Fernando, and A. Wells. A new approach to peak threshold estimation for impulsive noise reduction over power line fading channels. IEEE Syst. J., pages 1-12, Mar. 2018.

[28] Y. Wang, C. Yang, and B. Ai. Iterative companding transform and filtering for reducing PAPR of OFDM signal. IEEE Trans. Consum. Electron., 61(2):144-150, May 2015.
[29] Y. Wang, L. H. Wang, J. H. Ge, and B. Ai. An Efficient Nonlinear Companding Transform for Reducing PAPR of OFDM Signals. IEEE Trans. Broadcast., 58(4):677-684, Dec. 2012.

[30] N. Ali, R. Almahainy, A. Al-Shabili, N. Almoosa, and R. Abd-Alhameed. Analysis of improved $\mu$-law companding technique for OFDM systems. IEEE Trans. Consum. Electron., 63(2):126-134, May 2017.

[31] S. Peng, Y. Shen, and Z. Yuan. PAPR reduction of multi-carrier systems with simple nonlinear companding transform. Electron. Lett., 50(6):473475, Mar. 2014

[32] S. Jeng and J. Chen. Efficient PAPR reduction in OFDM systems based on a companding technique with trapezium distribution. IEEE Trans. Broadcast., 57(2):291-298, Jun. 2011.

[33] Y. Rahmatallah, N. Bouaynaya, and S. Mohan. Bit Error Rate Performance of Linear Companding Transforms for PAPR Reduction in OFDM Systems. In GLOBECOM 2011, pages 1-5, Dec. 2011.

[34] A. Mattsson, G. Mendenhall, and T. Dittmer. Comments on: "Reduction of peak-to-average power ratio of OFDM system using a companding technique". IEEE Trans. Broadcast., 45(4):418-419, Dec. 1999.

[35] K. Anoh, B. Adebisi, K. M. Rabie, and C. Tanriover. Root-based nonlinear companding technique for reducing PAPR of precoded OFDM signals. IEEE Access, 6:4618 - 4629, Dec. 2017.

[36] K. Anoh, R. Abd-Alhameed, Y. Dama, S. Jones, P. Pillai, and K. Voudouris. An Investigation of PMEPR of WPT-OFDM and OFDM Multicarrier Systems. J. Commun. Network. NWPJ-201307-07, 3(3):4552, 2013.

[37] W. Tranter, T. Rappaport, K. Kosbar, and K. Shanmugan. Principles of communication systems simulation with wireless applications, volume 1 . Prentice Hall New Jersey, 2004.

[38] Y. Rahmatallah, N. Bouaynaya, and S. Mohan. On the performance of linear and nonlinear companding transforms in OFDM systems. In 2011 Wireless Telecom. Symp. (WTS), pages 1-5, Apr. 2011.

[39] Y. Wang and Z. Luo. Optimized iterative clipping and filtering for PAPR reduction of OFDM signals. IEEE Trans. Commun., 59(1):33-37, Jan. 2011.

[40] P. Banelli. Non-linear transformations of Gaussians and Gaussianmixtures with implications on estimation and information theory. arXiv preprint arXiv:1111.5950v3, May 2013.

[41] R. Gangopadhyay and S. Ray. Performance Evaluation of OFDM in Nonlinear» Fading Channel Using PAPR Reduction Scheme and FEC. In NCC Conference. Citeseer, 2001.

[42] H. Gacanin and F. Adachi. On transmission performance of OFDM-based schemes using MMSE-FDE in a frequency-selective fading channel. EURASIP J. Wireless Commun. Netw., 2011(1):193, 2011.

[43] A. K. Gurung, F. S. Al-Qahtani, A. Z. Sadik, and Z. M. Hussain. OneIteration-Clipping-filtering (OICF) scheme for PAPR reduction of OFDM signals. In 2008 Intl. Conf. Advanced Technol. Commun., pages 207-210, Oct. 2008.

[44] Z. Kollár and P. Horváth. PAPR reduction of FBMC by clipping and its iterative compensation. J. Comput. Netw. Commun., 2012, May 2012.

[45] G. Primolevo, O. Simeone, and U. Spagnolini. Effects of imperfect channel state information on the capacity of broadcast OSDMA-MIMO systems. In IEEE 5th Workshop on Signal Process. Advanc. Wireless Commun., pages 546-550, 2004.

[46] B. He, X. Zhou, and T. Abhayapala. Wireless physical layer security with imperfect channel state information: A survey. arXiv preprint arXiv:1307.4146, 2013

[47] K. M. Rabie and E. Alsusae. On Improving Communication Robustness in PLC Systems for More Reliable Smart Grid Applications. IEEE Trans. Smart Grid, 6(6):2746-2756, Nov 2015. 